Zwischen Manganat(VI) und $\mathrm{MnO}_{2}$ einerseits und Manganat( $V$ ) andererseits besteht somit ein Gleichgewicht

$$
\stackrel{+6}{\mathrm{Na}_{2} \mathrm{MnO}_{4}}+\stackrel{+4}{\mathrm{MnO}_{2}}+\underset{4 \mathrm{NaOH}}{\rightleftharpoons} \underset{2 \mathrm{Na}_{3} \mathrm{MnO}_{4}}{+5}+2 \mathrm{H}_{2} \mathrm{O}
$$

das dem Massenwirkungsgesetz entsprechend bei hoher NaOH-Konzentration so weit nach rechts verschoben ist, daß die Löslichkeit des Manganat(V)-hydrats überschritten wird. Die genauere Festlegung der Gleichgewichte und der zugehörigen Redoxpotentiale in Abhängigkeit von $\mathrm{NaOH}-$ Konzentration und Temperatur bleibt einer späteren Mitteilung vorbehalten.

Die Darstellung von Manganat(V)-hydrat gelingt sehr einfach: Mit einer Lösung von $2 \mathrm{~g}$ feinst pulverisiertem $\mathrm{KMnO}_{4}$ in $50 \mathrm{ccm}$ Natronlauge $(1+2,5)$ werden $3,5 \mathrm{~g}$ fein zerriebenes $\mathrm{Na}_{2} \mathrm{SO}_{3}$ $+7 \mathrm{H}_{2} \mathrm{O}$ bei $0^{\circ}$ etwa $10 \mathrm{Min}$. verrührt, bis das Ganze einheitlich heliblau geworden ist. Dann saugt man scharf ab und wäscht mit Natronlauge $(1+2,5)$ nach.

Lösungen von Manganat(VII) oder Manganat (VI) in Natronlauge $(1+2,5)$ werden in der Kälte durch $\mathrm{Na}_{2} \mathrm{SO}_{3}, \mathrm{NaOOCH}$ oder $\mathrm{Na}_{2} \mathrm{~S}_{2} \mathrm{O}_{3}$ quantitativ bis zur 5-wertigen Stufe reduziert. Bei mehreren anderen Reduktionsmitteln tritt blaues Manganat(V) nur vorübergehend als $Z$ wischenstufe auf. Besonders hübsch sieht die Reaktion von $\mathrm{KMnO}_{4}$ mit $\mathrm{NaBO}_{2} \cdot \mathrm{H}_{2} \mathrm{O}_{2}$ aus; hier zeigen sich innerhalb von 1-2 Min. alle vier Wertigkeitsstufen des Man- gans (rotviolett - tiefgrün - himmelblau braungelb). Eingehende Versuche haben gezeigt, daß die fünfwertige Stufe des Mangans etwa ebenso häufig auftritt wie andere Wertigkeitsstufen, sofern man nur in stark alkalischer Lösung und in der Kälte arbeitet.

In dem dargestellten Manganat(V)-hydrat waren $\mathrm{SO}_{4}-2$ und $\mathrm{K}+$ nicht enthalten. Die jodometrische $\mathrm{Be}-$ stimmung des Oxydationswertes ergab 3,01 val/g-atom Mn entspr. der Wertigkeitsstufe 5,01. Zur Analyse wurde Mangan durch Ammoniak und Brom abgetrennt; Mangan wurde als $\mathrm{MnSO}_{4}$, Natrium als $\mathrm{Na}_{2} \mathrm{SO}_{4}$ gewogen. Bei zwei verschiedenen Präparaten ergaben sich folgende Werte:

$$
\begin{array}{rccc} 
& \mathrm{Na}_{2} \mathrm{O} & \mathrm{Mn}_{2} \mathrm{O}_{5} & \mathrm{H}_{2} \mathrm{O} \text { (Differenz) } \\
\text { I. } & 23,50 & 16,14 & 60,36 \text { Gew.- } \% \\
\text { II. } & 23,78 & 16,41 & 59,81 \text { Gew. } \%
\end{array}
$$

Beiden Präparaten haftete Natronlauge an, deren Gehalt zu 20,88 Gew.-\% $\mathrm{Na}_{2} \mathrm{O}$ bestimmt wurde. Die gefundenen Analysenwerte müssen, in ein Koordinatensystem eingetragen, auf einer Geraden liegen, welche die Punkte der Zusammensetzungen von Natronlauge und gesuchtem Hydrat miteinander verbindet. Beide Analysenwerte liégen nun recht genau auf der Verbindungslinie zum Punkt $\mathrm{Na}_{3} \mathrm{MnO}_{4}+10 \mathrm{H}_{2} \mathrm{O}$; theoretische Werte sind z. B. $23,7 \% \mathrm{Na}_{2} \mathrm{O}, 16,2 \% \mathrm{Mn}_{2} \mathrm{O}_{5}, 60,1 \% \mathrm{H}_{2} \mathrm{O}$. Für das $12-\mathrm{Hy}-$ drat, dessen Vorliegen zunächst vermutet worden war, sollte bei gleichem Mangangehalt gefunden werden: $22,4 \% \mathrm{Na}_{2} \mathrm{O}, 16,2 \% \mathrm{Mn}_{2} \mathrm{O}_{5}, 61,4 \% \mathrm{H}_{2} \mathrm{O}$. Wie man erwarten darf, scheint somit das 5-wertige Mangan dem 5-wertigen Vanadium besonders ähnlich zu sein, das ebenfalls Hydrate mit $10 \mathrm{H}_{2} \mathrm{O}$ bildet. Uber die Mischkristallbildung zwischen Natriummanganat(V) einerseits und tertiärem Natriumarsenat, -phosphat und -vanadat andererseits sowie über die Hydratstufen sind noch eingehendere Untersuchungen erforderlich.

\title{
Zuf Biochemie der Schilddrüsenfunktion, III. Mitteilung: Die entgiftende Wirkung des Vitamins C gegenüber kropferzeugenden Substanzen
}

\author{
Von Friedrich HÜter \\ Aus dem Institut für Chemotherapie „Georg-Speyer-Haus“, Frankfurt a. M. \\ (Z. Naturforschg. 1, 283-287 [1946]; eingegangen am 8. April 1946)

\begin{abstract}
Der Schilddrüsenreizstoff des Weißkohles erzeugt im lebenden Organismus einen relativen C-Vitaminmangel. Die Ascorbinsäure wirkt hinsichtlich strumigener Substanzen kropfhemmend. Das rasche Entstehen einer mehrfach vergrößerten, basedowähnlichen Schilddrüse durch eine pflanzliche Noxe plus Allylthioharnstoff wird beschrieben.
\end{abstract}

$\mathrm{M}$ a rine und Mitarbeiter ${ }^{2}$ berichten über einen kropfhemmenden Pflanzenwirkstoff und nehmen an, das Vitamin $\mathrm{C}$ übe vielleicht diese antagonistische Wirkung aus; sie bringen aber dafür

${ }^{1}$ I. Mitteilg.: Th. Wagner-Jauregg u. E. Schrei be r, Biochem. Z. 317, 21 [1944]; II. Mitteilg.: Th. Wa g ner-Jauregg u. J. Koch, unveröffentl. keine experimentellen Beweise. Nach ihren Befunden führt gedämpfter und abgepreßter Kohl zu einer stärkeren Schilddrüsenwucherung als frischer. Wir prüften mit handelsüblichem Weißkraut

$2 \mathrm{M}$ a r in e, B a u m a n , Webster u. Cipra, J. exp. Medicine 57, 121 [1933]; ferner J. biol. Chemistry 89, $213[1930]$. 
dieses Ergebnis nach und stellten Versuche mit Trockenweißkraut (TrWK.) an. F. Blu m${ }^{3}$ erwähnt eine Neutralisationskost, die Kohlkropfnahrung ausgleicht. Sie besteht aus Endiviensalat (Cichorium endivia L.), der mit Weißkohl im Verhältnis 1:1 verfüttert wird. Das biologisch wirksame, antistrumigene Prinzip des Salates besteht nach unserer Ansicht aus Jodalkali und Ascorbinsäure. Der Vitamin-C-Gehalt der Crucifere und der Composite zusammengenommen, reicht für die Entgiftung der Noxe aus, deshalb tritt kein Schilddrüsenschaden auf.

Bei Stepp, Kühnau u. Schroeder ${ }^{4}$ ist der.Vitamin-C-Gehalt in $100 \mathrm{~g}$ Endivie durch die Grenzwerte

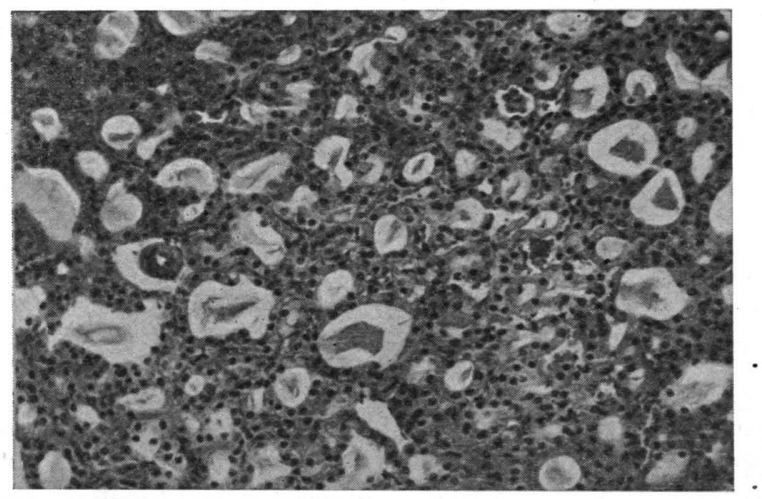

Abb. 1. Schilddrüsenschnitt von Kaninchen Nr. 460; Trockenweißkrauttier; Struma diffusa parenchymatosa. Vergr. 150/1.

3-10 mg und den Mittelwert $5 \mathrm{mg}$ tabellarisch festgelegt. Die Ascorbinsäuremenge in $100 \mathrm{~g}$ gekochtem Weißkohlgemüse soll einen Wert von 5-10 mg erreichen. Nach $\mathrm{V}$ a j i $\mathrm{c}^{5}$ beträgt der Vitamin-C-Gehalt in $1 \mathrm{~kg}$ frischem Weißkraut 20-50 mg; durch das Kochen tritt ein Vitaminverlust von $50 \%$ ein; Lagerung beeinträchtigt den Ascorbinsäuregehalt des Krautes in geringem Maße.

Es ist bekannt, daß die pflanzliche Kropfnoxe aller Wahrscheinlichkeit nach ein bislang noch unbekanntes Thioglykosid - durch den einstündigen Kochvorgang nicht zerstört und nur der Ascorbinsäurewert vermindert wird. Die kropferzeugende Substanz übersteht das Kochen vermutlich besser als der neutralisierende Wirkstoff. Eine Ascorbinsäuremenge, wie sie neben dem schwefelhaltigen Reizstoff in der Krautnahrung vorkommt genügt offenbar nicht, um die Noxe zu entgiften.

3 Schweiz. med. Wschr. 73, 1046 [1943].

4 Siehe Leitfaden „Die Vitamine und ihre klinische Anwendung", 6. Aufl. 1944, S. 468.

5 B. V aji ic, Biochem. Z. 309, 343 [1941].
Es liegt demnach wahrscheinlich ein unterschwelliger Wert des Vitamins C vor. Auch im TrWK. ist wohl der Wirkstoffgehalt unzureichend. So vermögen also Weißkraut wie auch Trockenkraut bei längerer einseitiger Verabreichung am Kaninchen eine Präbasedow-Thyreoidea zu erzeugen. Aus den unten beschriebenen Versuchen erhellt, daß der Kohlkropf durch hohe Vitamin-C-Zulage zum Weißkrautfutter verhütet wird. Synthetischer $A l$ lylthioharnstoff ${ }^{6}, \mathrm{H}_{2} \mathrm{C}: \mathrm{CH} . \mathrm{CH}_{2} \cdot \mathrm{NH} \cdot \mathrm{CS} \cdot \mathrm{NH}_{2}$, wurde wegen seiner Fähigkeit, histologisch basedowartige Schilddrüsen zu erzeugen ${ }^{1}$ außer dem natürlichen, kropferzeugenden Agens bei unseren Untersuchungen verwandt. Eine bestimmte Ascor-

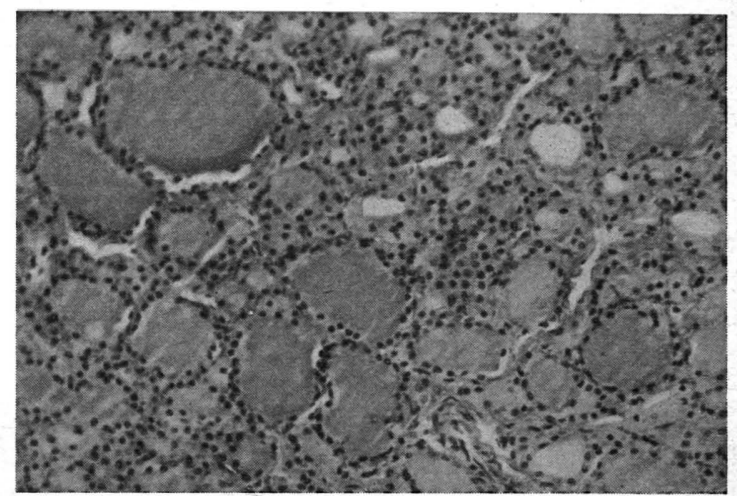

Abb. 2. Schnitt einer angenähert normalen Kaninchenschilddrüse Nr. 457; mit Vitamin C behandeltes Trockenweißkrauttier. Vergr. 150/1.

binsäuremenge als Futterbeilage bremst die schilddrüsenschädigende Wirkung des ungesättigten Thioharnstoffes, den man Kaninchen parenteral zuführt. Es wird schließlich noch gezeigt, wie das strumigene Agens von verfüttertem TrWK. und von gleichzeitig injiciertem Sinamin (Allylthioharnstoff) $\mathrm{zu}$ einer verstärkten Verkropfung führen.

Wir fütterten ein französisches Silberkaninchen (Tab. 1, Nr. 435) 50 Tage hindurch mit frischem, 1 Stde. gekochtem Weißkraut. Man fand bei dem Sezieren eine etwas vergrößerte, jodfreie Schilddrüse (SD.). Sie wies, mikroskkopisch gesehen, ein Präbasedow-Stadium ${ }^{7}$ auf. Ein Bild, ähnlich einer Struma diffusa parenchymatosa des Menschen ${ }^{8}$, zeigte das Chinchillatier Nr. 460, welches

6 Darstellungsweise in Beilsteins Handbuch d. Organ. Chemie, Bd. IV, S. 211.

7 F. B l u m, Endokrinologie 19, 19 [1937].

$8 \mathrm{C}$. Wegelin in Henke-Lubarsch, Handbuch d. spez. pathologischen Anatomie u. Histologie, Bd. VIII, S. 156, Springer-Verlag, Berlin 1926. 


\begin{tabular}{|c|c|c|c|c|c|c|c|c|c|c|c|c|c|c|c|}
\hline \multirow[b]{2}{*}{$\begin{array}{c}\text { Tier } \\
\text { Nr. }\end{array}$} & \multirow[b]{2}{*}{ 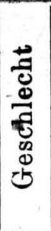 } & \multirow[b]{2}{*}{ Rasse } & \multirow[b]{2}{*}{$\begin{array}{c}\text { Insges. verabreichte } \\
\text { natürl. bzw. chem. } \\
\text { Noxe } \\
\text { in Tagen }\end{array}$} & \multirow[b]{2}{*}{ 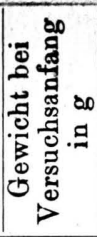 } & \multirow[b]{2}{*}{ 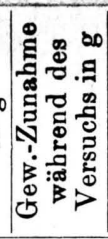 } & \multirow[b]{2}{*}{ 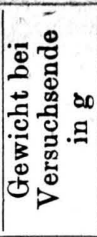 } & \multicolumn{4}{|c|}{ Lo,ber } & \multicolumn{4}{|c|}{ Schilddrüse } & \multirow[b]{2}{*}{ Histologischer SD.-Befund } \\
\hline & & & & & & & 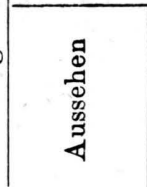 & 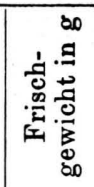 & 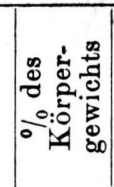 & 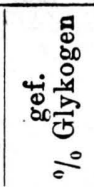 & 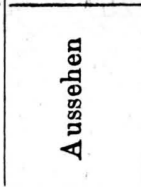 & 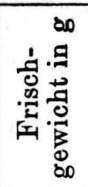 & 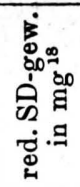 & 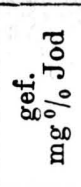 & \\
\hline 435 & $0^{7}$ & $\begin{array}{l}\text { Französ. } \\
\text { Silber- } \\
\text { kaninchen }\end{array}$ & $\begin{array}{l}50 \mathrm{~kg} \text { frisches ge- } \\
\text { kochtesWeißkraut, } \\
50 \text { Tage (10.8.- } \\
29.9 .1944)\end{array}$ & 1540 & 680 & 2220 & normal & 91,5 & 4,1 & 13,83 & rot & 0,18 & 82 & 0 & $\begin{array}{l}\text { stark gewuchertes Epithel; } \\
\text { fast völliger Kolloid- } \\
\text { schwund. Praebasedow; } \\
\text { vergl. E. Maschmann }{ }^{10} \text {, } \\
\text { Abb. 4. }\end{array}$ \\
\hline 460 & $\sigma^{7}$ & $\begin{array}{l}\text { Klein- } \\
\text { Chinchilla } \\
\end{array}$ & \begin{tabular}{|l|} 
7,28 kg Trocken- \\
weißkraut ${ }^{16}$ \\
91 Tage (29. $8 .-$ \\
28. 11.1945)
\end{tabular} & $1 ; 20$ & 880 & 2600 & hellbraun & 106 & 4,08 & 10,42 & $\begin{array}{l}\text { rot- } \\
\text { sattrot }\end{array}$ & 0,21 & 80 & 0 & $\begin{array}{l}\text { Drüsé in Läppchen gefel- } \\
\text { dert; epitheliale Zellver- } \\
\text { mehrung; Bläschen mit } \\
\text { Kolloidschollen; diffus } \\
\text { parenchymatöse Struma; } \\
\text { vergl. Abb. 1. }\end{array}$ \\
\hline 461 & o & $\begin{array}{l}\text { Klein- } \\
\text { Chinchilla }\end{array}$ & $\begin{array}{l}\text { 7,28 } \mathrm{kg} \text { Trocken- } \\
\text { weißkraut, } \\
\text { 1,925 g Vit. C, } \\
\text { 91 Tage }(29.8 .- \\
\text { 28. 11. } 1945)\end{array}$ & 1690 & 980 & 2670 & graubraun & 112 & 4,2 & 11,62 & $\begin{array}{l}\text { bla } \beta \\
\text { mit roten } \\
\text { Spitzen }\end{array}$ & 0,15 & 56 & 6,56 & $\begin{array}{l}\text { z.Tl.kleinfollikuläre Hyper- } \\
\text { plasie; sonst natürlicher } \\
\text { Zellaufbau. }\end{array}$ \\
\hline 457 & 우 & $\begin{array}{l}\text { Klein- } \\
\text { Chinchilla }\end{array}$ & $\begin{array}{l}9,6 \mathrm{~kg} \text { Trocken- } \\
\text { weißkraut, } \\
2,575 \mathrm{~g} \text { Vit. } \mathrm{C}^{17} \text {, } \\
\text { 120 Tage }(30.5 .- \\
\text { 28. 8. } 1945)\end{array}$ & 2500 & 150 & 2650 & normal & 75,5 & 2,92 & 6,58 & blaßrot & 0,12 & 46 & 9,28 & $\begin{array}{l}\text { mit eosinophilem Kolloid } \\
\text { gefüllte, große Follikel; } \\
\text { ziemlich normale SD. } \\
\text { Abb. } 2 .\end{array}$ \\
\hline 463 & $0^{7}$ & $\begin{array}{l}\text { Blauer } \\
\text { Wiener }\end{array}$ & $\begin{array}{l}\text { 7,8 g Allylthioharn- } \\
\text { stoff (subcutan), } \\
\text { 1,3 Vit. C (per os) } \\
\text { 91 Tage (5. 9.- } \\
\text { 5. 12. 1945) }\end{array}$ & 2240 & 380 & 2620 & $\begin{array}{l}\text { schoko- } \\
\text { laden- } \\
\text { braun }\end{array}$ & 85,5 & 3,26 & 4,24 & blaßrot & 0,21 & 80 & 0 & $\begin{array}{l}\text { hyperämisch; deformierte } \\
\text { Zellen; interfollikuläre, } \\
\text { weite Bahnen; Kolloid- } \\
\text { speicherung. Vielleicht } \\
\text { beginnende, aber noch } \\
\text { wenig ausgesprochene } \\
\text { Ausschwemmung. }\end{array}$ \\
\hline 453 & $0^{7}$ & $\begin{array}{l}\text { hellblau- } \\
\text { grau } \\
\text { Fehkan. }\end{array}$ & $\begin{array}{c}\text { 5,2 g Allylthioharnst. } \\
\text { (subcutan), 1,5 kg } \\
\text { Trockenweißkraut } \\
\text { (peroral), 60 Tage } \\
\text { (13.1.-14.3.1945) }\end{array}$ & 2350 & 40 & 2390 & $\begin{array}{l}\text { dunkel- } \\
\text { braun - } \\
\text { schwärz- } \\
\text { lich }\end{array}$ & 70,55 & 2,95 & 0,60 & rot & 1,64 & 686 & 0 & $\begin{array}{l}\text { kolloidfreie, regellos ge- } \\
\text { formte Follikel; hohe } \\
\text { wabige Epithelzellen; } \\
\text { sehr aktive SD. Abb. } 3 .\end{array}$ \\
\hline
\end{tabular}

Tab. 1. Versuchskaninchen.

Tägliches Grundfutter: $10 \mathrm{~g}$ Hafer, etwas Heu und bidestilliertes Wasser nach Belieben; Kaninchen Nr. 463 fraß außerdem Dickwurz und Kartoffelschalen (Normalkost). - Glykogenbestimmung nach P flüg er ; Jodanalyse-Verfahren nach B lu m ${ }^{14}$.

14 Schweiz. med. Wschr. 71, 841 [1941].

15 Geschwistertiere, von Häsin Nr. 457 geworfen.

16 Herstellende Firma: Helvetia, Konservenfabrik Groß-Gerau.
17 Natriumsalz der l-Ascorbinsäure (jodfrei); Merck-Präparat.

18 Das auf $1 \mathrm{~kg}$ Körpergewicht reduzierte Schilddrüsengewicht normaler, noxenfrei ernährter Kaninchen beträgt bei unserem Tiermaterial etwa 40 bis $60 \mathrm{mg}$. 
über 3 Monate ein 4 Jahre altes Trockenweißkraut als Futter erhielt (Abb. 1). Dieser Tierversuch ergab, daß selbst eine Lagerung von 4 Jahren den kropfhervorrufenden Stoff nicht zerstörte. Durch eine chemische Methode wurde nachgewiesen, daß auch noch Vitamin C im benutzten TrWK. vorhanden war, und zwar wurden $16,5 \mathrm{mg}$ Vitamin $\mathrm{C}$ in $100 \mathrm{~g}$ Trockensubstanz gefunden $^{9}$. Die quantitative Vitamin-C-Bestimmung bestätigte für das TrWK. die Ansicht von V a ji c ${ }^{4}$, wonach das C-Vitamin im Weißkraut sehr stabil ist. Unsere beiden vorher erwähnten Versuche sehen wir als Beweis dafür an, daß der Ascorbinsäuregehalt des gekochten Weißkrautes wie des TrKW. zu klein ist, um die Wirkung des vermuteten Thioglykosids aufzuheben. Zwei weitere Kaninchenversuche wurden mit Trockenkraut und Vitamin-C-Beilage ausgeführt. Man tränkte täglich die Krautration, $80 \mathrm{~g}$, mit $1 \mathrm{ccm}$ einer 2,5-proz. wäßrigen

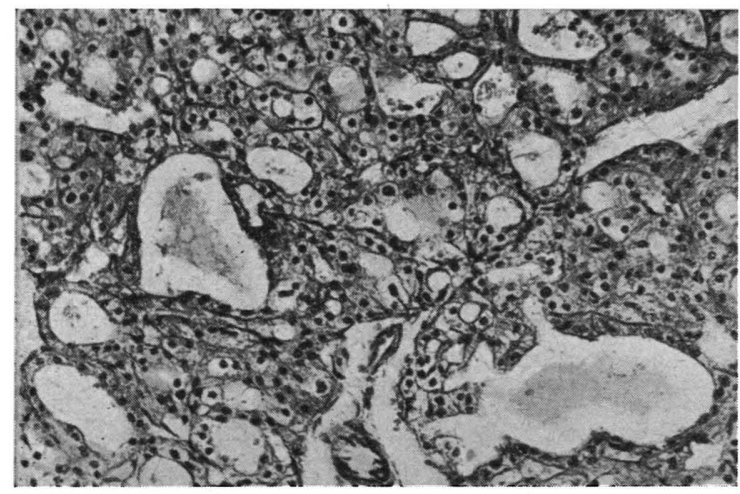

Abb. 3. Histologischer Schnitt der basedowähnlich entarteten Schilddrüse von Kaninchen Nr. 453; mit Allylthioharnstoff injiciertes Trockenweißkrauttier. Vergr. 150/1.

Vitamin-C-Natriumsalz-Lösung. Das Jungtier Nr. 461 besaß nach 91 Versuchstagen eine Schilddrüse, deren Größe und Jodgehalt der Norm entsprach (red. SD.-Gewicht $^{18} 56 \mathrm{mg}$ ). Der histologische Schnitt bot kleine Follikel und teilweise Epithelwucherung. Sonst traten keine Unterschiede der SD. zu der des Muttertieres Nr. 457 auf. Dieses ausgewachsene Kaninchen hatte nach mehrmonatiger Verfütterung von $9,6 \mathrm{~kg}$ TrWK. unter Zugabe von $2,575 \mathrm{~g}$ Vitamin $\mathrm{C}$ eine $\mathrm{SD}$. mit normalem Strukturbild ${ }^{\mathbf{1 0}}$ (Abb. 2).

An einem Blauen Wiener (Tab. 1, Nr. 463) wurde die Hemmung der strumigenen Kraft von Allylthioharnstoff durch Vitamin C geprüft. Er bekam sechsmal pro Woche $100 \mathrm{mg}$ chemisch reines Sinamin, in $1 \mathrm{ccm}$ Wasser aufgelöst, subcutan zugeführt und erhielt täglich $25 \mathrm{mg}$ Ascorbinsäure (Na-Salz) dem Körnerfutter zugesetzt. 1/4 Jahr später stellten wir eine schwach vergrößerte und

9 Für die Analyse danke ich bestens Hrn. Prof. Dr. Dr. W. D i ema i r, Direktor des Universitätsinstituts f. Nahrungsmittelchemie, Frankfurt a. M.

10 Nähere Beschreibung der normalen Drüse bei E. Ma s chm ann, Naturwiss. 30, 262 [1942]. blutreiche Kaninchenschilddrüse (red. SD.-Gewicht $80 \mathrm{mg}$ ) fest. Jod war in ihr analytisch nicht nachweisbar. Zwischen den deformierten Follikeln waren Spalten zu sehen. Nach Th. Wa g n e r -J a u reg g und J. K o c h ${ }^{\mathbf{1 1}}$ beträgt das reduzierte Schilddrüsengewicht von Versuchskaninchen, die mit der gleichen Allylthioharnstoffdosis behandelt waren, 140-160 mg. Die hier eben noch vorliegende Hypertrophie ist vielleicht dem ungenügenden Ascorbinsäureangebot zuzuschreiben, so daß die Entgiftung nicht quantitativ war.

Das Zusammenwirken verschiedener SD.-Schädigungsfaktoren zeigt sich deutlich in folgendem Experiment. Es wurde einem täglich $80 \mathrm{~g}$ Trockenweißkraut fressenden Tier pro die $100 \mathrm{mg}$ Allylthioharnstoff injiciert. Auf diese Weise konnte die Entartung der SD. nicht nur verhindert, sondern noch gesteigert werden. Die Sektion des Kaninchens Nr. 453 (Tab. 1) zeigte nach $7^{\mathbf{1}} / \mathbf{2}^{-}$ wöchiger Versuchsdauer eine durch Vergrößerung und Hyperämie ausgezeichnete SD. Ihr histologisches Aussehen ist durch Abb. 3 wiedergegeben. Das reduzierte Schilddrüsengewicht betrug $686 \mathrm{mg}$, demnach mehr als das 10-fache der Norm. Die pflanzliche Noxe im TrWK. und das synthetische Sinamin, beide gleichzeitig peroral bzw. subcutan verabreicht, wirkten also mit erhöhter Macht kropferzeugend. Die dunkelbraune bis schwärzliche Farbe der Leber und ihr geringer Glykogengehalt $(0,6 \%)$ deuteten eine Leberschädigung an, wie dies schon früher in einem Fall von Allylthioharnstoff-Injektion beobachtet worden war ${ }^{1}$. Der Gewichtssturz, der fast immer bei den mit Allylthioharnstoff gespritzten Tieren auftrat ${ }^{11}$, wurde durch das bekömmliche Trockenweißkrautfutter vermieden.

Wie hat man sich wohl den Entgiftungsverlauf kropferregender Substanzen im tierischen Organismus vorzustellen? Die aus dem Pflanzenreich stammenden sowie die chemischen Reizstoffe schädigen u. a. die Leber. Der Vitamin-C-Vorrat dieses Organs wird durch die Noxeneinwirkung verbraucht, es tritt zumindest eine Verarmung an diesem Wirkstoff auf. Durch zusätzliche Vitamin-CApplikation wird die Leber entlastet, mit neuem C-Faktor aufgefüllt, aktiviert und wieder in den Normalzustand versetzt. Außerdem haben wir von dem Antagonismus der $l$-Ascorbinsäure gegenüber dem Glykogenabbau in der Leber, der unter Umständen durch Reizstoffe gesteigert werden kann, Kenntnis erhalten ${ }^{12}$.

Man kann hinsichtlich unserer aufgezeigten Befunde die Ascorbinsäure als einen Leberschutzstoff betrachten, da sie zum Abschwächen SD.schädigender Mittel benutzt wird. Ob aber durch die einseitige Kohlkropfkost eine allgemeine rela-

11 Unveröffentlichte Versuche aus der Biochem. Abtlg. des ,Georg-Speyer“-Hauses.

${ }_{12}$ Vergl. S. Thadd ea u. H. J. Runne, Z. exp. Medizin 103, 283 [1938]. 
tive Avitaminose ${ }^{13}$ erzeugt wird, das werden spätere Untersuchungen kıären.

Zum Schluß sei auf folgendes aufmerksam gemacht: Kohlgemüse ist ein Bestandteil der menschlichen Nahrung und könnte bei zu reichlichem Genuß als Kropfnoxe dann eine Rolle spielen, wenn die kropferregende Substanz weder durch eine Vitamin C enthaltende Kost noch durch ein Ascorbinsäure-Präparat entgiftet wird. Bei der absichtlichen Erzeugung von experimentellen Schilddrüsenveränderungen ist es wichtig, zu beachten, daß mit dem Futter nicht übermäßig C-Vitamin,

13 Über die Deutung des Begriffes s. Klaus Schw a r z, Hoppe-Seylers Z. physiol. Chem. 281, 101 [1944]. im Hinblick auf dessen antistrumigenen Eigenschaften, verabreicht werden sollte.. Sicherlich wirkt auch vitamin-C-reiche Kost bei Mensch und Tier prophylaktisch gegen Kohlkropf. Eine Prophylaxe mit Ascorbinsäure ist in diesem Falle der mit Alkalijodid (wegen der Gefahr der Basedow-Entwicklung) vorzuziehen. Das Suchen nach Strumen hemmenden und erregenden Stoffen wird fortgesetzt, um in kropfartige Krankheitsvorgänge einen besseren Einblick als bisher zu bekommen.

Hrn. Prof. Dr. A. Dietrich, Stuttgart, danken wir herzlich für die Beurteilung einiger histologischer Präparate. Frl. E. Schreiber war an der Durchführung der Versuche mitbeteiligt. Hr. Ma a führte die mikrophotographischen Aufnahmen aus.

\title{
Zur Entwicklungsphysiologie der Selbststerilität von Petunia
}

\author{
Von Josef Straub \\ Aus dem Kaiser-Wilhelm-Institut für Biologie, Abtlg. von Wettstein, Hechingen-Boll \\ (Z. Naturforschg. 1, 287-291 [1946]; eingegangen am 22. März 1946) \\ Die Vorstellung Y as udas über die Selbststerilität bei Petunia, wonach ein „Hemm- \\ stoff" vom Fruchtknoten nach oben in das Griffelgewebe diffundiere und die Wachstumsver- \\ langsamung der eigenen Pollenschläuche hervorrufe, ist nicht zutreffend. Durch Bestäu- \\ bungen in verschiedenen Höhen des Griffels wird bewiesen, das alle Teile des Griffel-Leit- \\ gewebes die Wachstumsreaktion mit den Pollenschläuchen in gleicher Weise auszuüben ver- \\ mögen. Das Wachstum der Pollenschläuche nach Selbstung hört stets nach $15-20 \mathrm{~mm}$ auf, \\ ganz gleich, wo und in welcher Richtung diese Strecke im Griffel zurückgelegt wird. Die \\ Wirkung des Mechanismus, welcher die Unterschiede im Schlauchwachstum nach Selbstung \\ und Fremdung bedingt, kann in vitro reproduziert werden. Läßt man nämlich aus dem abge- \\ schnittenen Ende eines zuvor bestäubten Griffels die Pollenschläuche in einen Tropfen \\ White-Lösung treten, so wachsen sie nach Zusatz fremden Leitgewebes besser als nach \\ Zusatz von eigenem.
}

Die Versuche wurden im Sommer und Herbst 1945 durchgeführt.

$\mathrm{D}_{\mathrm{s}}^{\mathrm{i}}$ e vorliegenden Untersuchungen an Petunia gehen auf einen Befund zurück, den wir bei Antirrhinum-Wildarten machten. Das diploide $A n$ tirrhinum glutinosum Wildsippe orgiva ist streng selbststeril. Die daraus ausgelösten Tetraploiden erwiesen sich als selbstfertil ${ }^{1}$. Im Gegensatz zu den Verhältnissen bei den Diploiden wachsen die Pollenschläuche der Tetraploiden bis zu den Eizellen durch, wo es zur Befruchtung und normalen Samenbildung kommt. Da das Wachstum des Pollenschlauches nicht selbständig, sondern vom Leitgewebe des Griffels abhängig ist, können Änderungen in seiner Intensität auf Änderungen der Pollenkonstitution oder solchen des Griffelgewebes beruhen. Wenn also diploide Pollenkörner in tetraploiden Griffeln besser wachsen als haploide Pol-

1 J. Stra u b, Ber. dtsch. bot. Ges. 59, 296 [1941]. lenkörner derselben Pflanze in den dazugehörigen diploiden Griffeln, so können dafür zwei bzw. drei verschiedene Ursachen angenommen werden. Entweder reagieren die diploiden Pollenkörner auf die gleichgebliebene Wachstumskomponente des Leitgewebes anders als die haploiden Körner, oder die Wachstumskomponente des Griffels hat sich verändert. Schließlich ist an eine Kombination beider Möglichkeiten zu denken. Zur Entscheidung ist es notwendig, von den selbststerilen Pflanzen homozygote Formen herzustellen und an ihnen entsprechende Kreuzungsexperimente durchzuführen. Diese Experimente sind im Gange. Daneben versuchen wir, die Wachstumsbedingungen der Pollenschläuche zu erfassen. Für diesen $\mathrm{Z}_{\text {weck }}$ sind Antirrhinum-Arten aus verschiedenen Gründen ungeeignet. Günstiger erscheint Petunia. Auch bei 